\title{
Radio Propagation Models in Wireless NETWORKS OF UNMANNED AERIAL VEHICLES
}

\author{
Jun Peng \\ Electrical Engineering Department \\ University of Texas - Rio Grande Valley \\ Edinburg, Texas, USA
}

\begin{abstract}
Although unmanned aerial vehicles (UAVs) were mostly studied and used for military purposes before, they have become very popular recently for both civil uses, such as law enforcement and crop survey, and for potential commercial uses such as grocery delivery and Internet extension. Researchers investigating new networking protocols for UAV networks usually need the help of simulations to test their protocol designs, particularly when networks of large scales are desired in their tests. One choice that researchers need to make in the simulation of UAV networks is the radio propagation model for the air links. In this paper we compare the three radio propagation models that are available in the ns 2 network simulation package and investigate if the choice of one particular model would have a significant impact on the simulation results for UAV networks.
\end{abstract}

\section{KEYWORDS}

Radio propagation models, unmanned aerial vehicles (UAV), network simulation, protocols

\section{INTRODUCTION}

As hardware and software become more advanced but even cheaper, unmanned aerial vehicles have evolved from being used only in military applications to being used in both civil and commercial applications. For example, people enthusiastic about photographing started to use UAVs to take videos or pictures of subjects from perspectives that they could not imagine or reach before due to the mounting costs of renting a plane or helicopter. Besides costs, it is much safer, faster and more environmentally friendly to use an UAV instead of a plane or helicopter in such an application. UAVs have also been used for law enforcement, surveillance, search and rescue, scientific research, and other applications [1].

As UAVs are used in more and more civil and commercial applications and become more and more popular, it will become inevitable that UAVs need to communicate with each other for purposes of coordination and collaborations. For example, UAVs will likely need some type of coordination in the future to avoid collisions among them. Besides, applications that require a large amount of UAVs working together dynamically, such as surveilling a large geographical area without interruption, also need the UAVs to be able to communicate with each other timely and effectively.

To enable UAVs to network themselves and communication with each other, researchers have been investigating topics at various layers of UAV networks. Asadpour et al. [2] studied the performance of IEEE 802.11n interfaces in UAV networks and they found that $802.11 \mathrm{n}$ performed poorly in highly mobile scenarios. Cai et cal. [3] proposed a medium access control 
(MAC) protocol for UAV ad-hoc networks with full-duplex radios. They proposed a token-based technique to update information in the network for efficiently handling the high mobility of UAVs. Ollero et al. [4] presented a platform that enables autonomous aerial vehicles to cooperate with ground wireless sensor-actuator networks. Alshbatat et al. [5] used the approach of cross layer design to deal with the high dynamics in UAV networks. Alshbatat et al. [6] also proposed an adaptive medium access control protocol for UAV networks using directional antennas. Gu et al. [7] proposed a routing scheme for multi-layer ad hoc networks that use UAVs in their backbones. Gu et al. [8] also presented a multiple access protocol for such type of network.

In summary, researchers have been actively investigating all types of topics in UAV networks. When UAV networks of large scales are needed in testing communication and networking protocols, simulations are usually the only practical method that would be available to most investigators. One important element in simulating an UAV network is the radio propagation model used in the network. There are three radio propagation models that are available in the ns- 2 network simulation platform. Although there are studies on some of these radio propagation models for vehicular networks [2], the studies focused on terrestrial networks. In this paper we compare the three models for UAV networks and study if the choice of a particular model would have a significant impact on simulation results.

The rest of the paper is organized as follows. Section 2 introduces the three radio propagation models that are available in the ns- 2 simulation platform. Section 3 presents our simulation results comparing these three models. Section 4 summarizes our findings.

\section{THE THREE RADIO PROPAGATION MODELS IN NS2}

\subsection{The Free Space Propagation Model}

The free space radio propagation model assumes, as its' name indicates, that the transmitter and the receiver are in free space without obstacles. The propagation formula is as follows [9]:

$$
\frac{P_{r}}{P_{t}}=\frac{A_{r} A_{t}}{d^{2} \lambda^{2}}
$$

where $P_{r}$ is the signal power at the receiving antenna, $P_{t}$ is the signal power at the transmitting antenna, $A_{r}$ is the effective area of the receiving antenna, $A_{t}$ is the effective area of the transmitting antenna, $d$ is the distance between the transmitting antenna and the receiving antenna, and $\lambda$ is the wavelength of the signal.

When we have an isotropic antenna, the effective area is $\lambda / 4 \pi$. Equation 1 thus becomes:

$$
\frac{P_{r}}{P_{t}}=\frac{\lambda^{2}}{(4 \pi d)^{2}}
$$

In a general case where we have an antenna gain that is not 1 for both the transmitting antenna and the receiving antenna, Equation 1 becomes: 


$$
\frac{P_{r}}{P_{t}}=G_{t} G_{r} \frac{\lambda^{2}}{(4 \pi d)^{2}}
$$

where $G_{t}$ is the antenna gain of the transmitting antenna and $G_{r}$ is the antenna gain of the receiving antenna.

Note that Equations 1 to 3 only apply, in general, under the following condition [9]:

$$
d \geq \frac{2 a^{2}}{\lambda}
$$

where $a$ is the largest linear dimension of either of the antennas.

\subsection{The Two-Ray Ground Propagation Model}

The two-ray ground propagation model [10] assumes that there are two signal paths from the transmitter to the receiver. One is the direct line-of-sight path while the other is the reflection path from the ground. The formula is as follows:

$$
\frac{P_{r}}{P_{t}}=G_{t} G_{r} \frac{h_{t}^{2} h_{r}^{2}}{d^{4}}
$$

where $h_{t}$ is the height of the transmitting antenna and $h_{r}$ is the height of the receiving antenna.

Note that Equation 5 applies under the following condition:

$$
d \geq \frac{4 \pi h_{t} h_{r}}{\lambda}
$$

\subsection{The Shadowing Model}

Due to multipath propagation effects, the received power at a receiving antenna is not a deterministic function of the communication distance as shown in the two models introduced earlier. Instead, the received power fluctuates as a random variable. The shadowing model [10] better reflects this characteristic of the received signal power than the other two models introduced earlier. The path loss is usually expressed in $\mathrm{dB}$ as follows in the the shadowing model:

$$
\left[P_{r}(d)-P_{r}\left(d_{0}\right)\right]_{d B}=-10 \beta \log \left(\frac{d}{d_{0}}\right)+X_{d B}
$$

where $d_{0}$ is a reference distance, $\beta$ is the path loss exponent, $X_{d B}$ is a Gaussian random variable having a zero mean and a standard deviation of $\delta_{d B}$. The typical values of $\beta$ and $\delta$ are shown in Tables 1 and 2, respectively. 
Table 1 Typical value of $\beta$ in different environment

\begin{tabular}{|c|c|}
\hline Environment & $\beta$ \\
\hline Outdoor, free space & 2 \\
\hline Outdoor, shadowed urban area & $2.7-5$ \\
\hline In building, line-of-sight & $1.6-1.8$ \\
\hline In building, obstructed & $4-6$ \\
\hline
\end{tabular}

Table 1 Typical value of $\delta$ in different environment

\begin{tabular}{|c|c|}
\hline Environment & $\delta$ \\
\hline Outdoor & $4-12$ \\
\hline Office, hard partition & 7 \\
\hline Office, soft partition & 9.6 \\
\hline Factory, line-of-sight & $3-6$ \\
\hline Factory, obstructed & 6.8 \\
\hline
\end{tabular}

\section{SIMULATION RESULTS}

After introducing the three radio propagation models that are available in ns2 in the previous section, in this section we investigate these three models for UAV networks and show simulation results. The simulation package ns 2 does not support simulations of three dimensional networks. We modified the package for UAV networks.

The simulated test bed is shown in Figure 1. The test bed has the same size for its width, length, and height, which is 500 meters. UAVs can fly freely in this defined space following the random waypoint model. The UAVs in the test bed use the IEEE 802.11 air interface that uses the default settings in the simulator. Each UAV sets up a CBR connection with another UAV at a random time during each simulation. A CBR source sends out packets at a rate of 10 packets per second and each packet has a size of 512 bytes. The routing protocol used in the test bed is DSR [11].

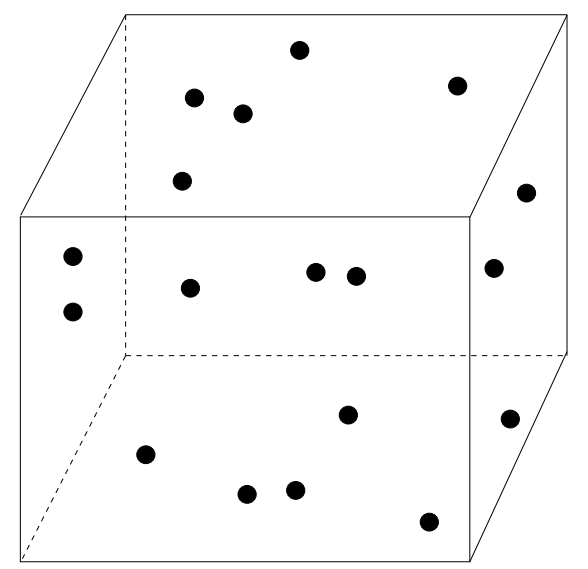

Figure 1 The simulated test bed 
In our simulations we tested the three radio propagation models with varying node densities in the test bed. Particularly, we varied the number of nodes in the test bed from 10 to 50 in our simulations of UAV networks using each model. In each simulation we collected data to calculate the average throughput and the average packet loss rate of the CBR flows in the network. We also collected data on the packet delay distributions in the network. Our simulation results are shown in Figures 2 to 6.

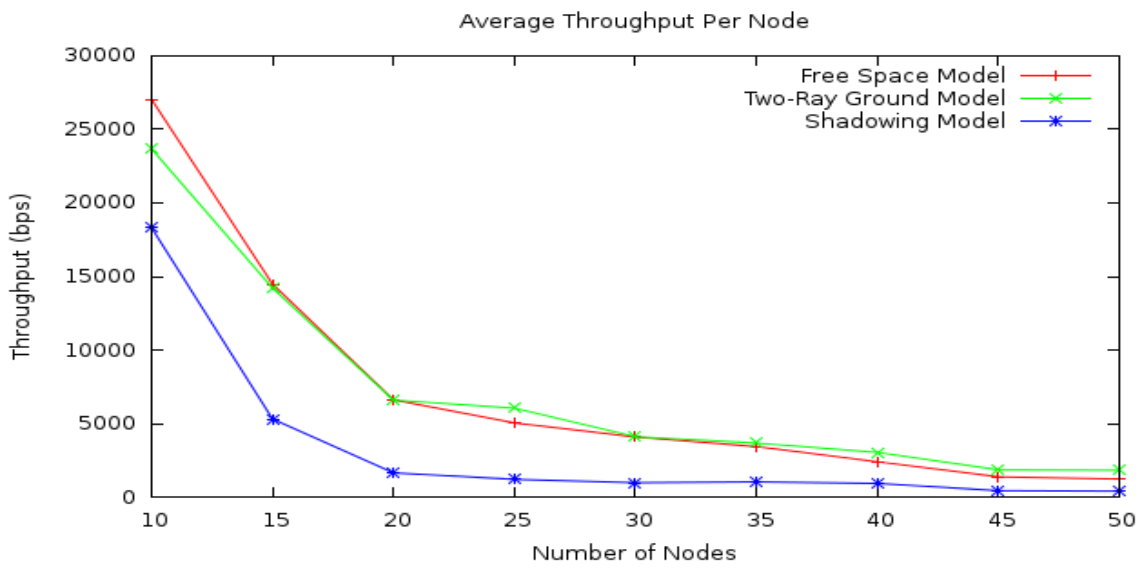

Figure 2. The average flow rate for the three propagation models

As shown in Figure 2, the average flow throughput in the network decreases as the node density increases in the network. This decrease of flow throughput is mainly caused by two factors. One is that the total traffic rate is higher in the network when there are more nodes in the network because each node starts a CBR flow at a random time in each simulation. The other factor is that the contention at the medium access control (MAC) layer is also higher when there are more nodes in the network. The throughput results are also corroborated by the packet loss rate shown in Figure 3. As shown in Figure 3, a higher node density in the network results in a higher packet loss rate in the network.

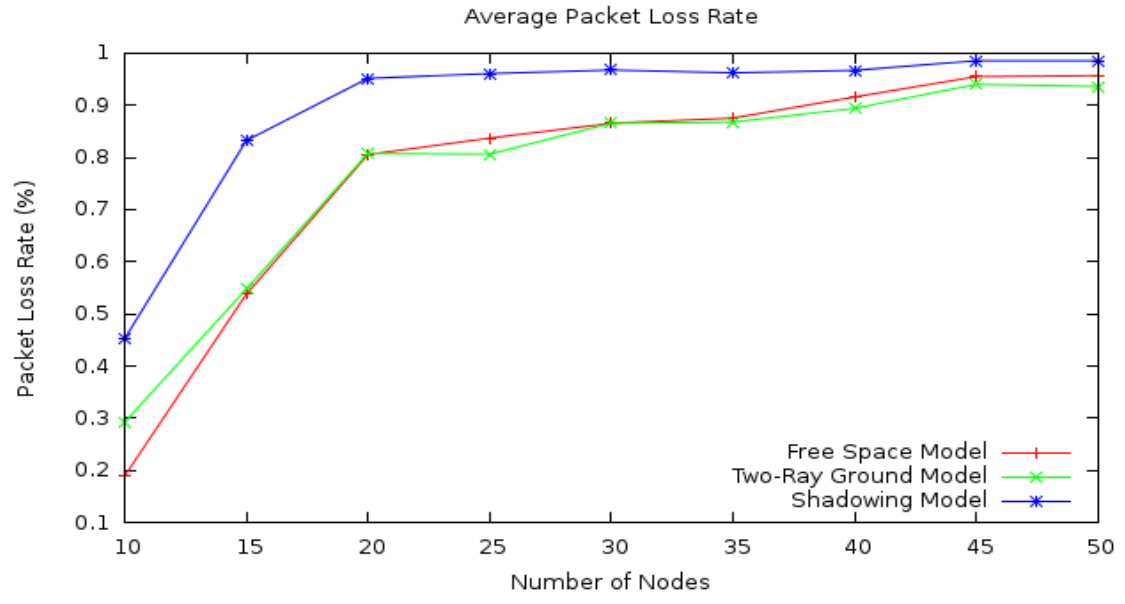

Figure 3. The average packet loss rate for the three propagation models 


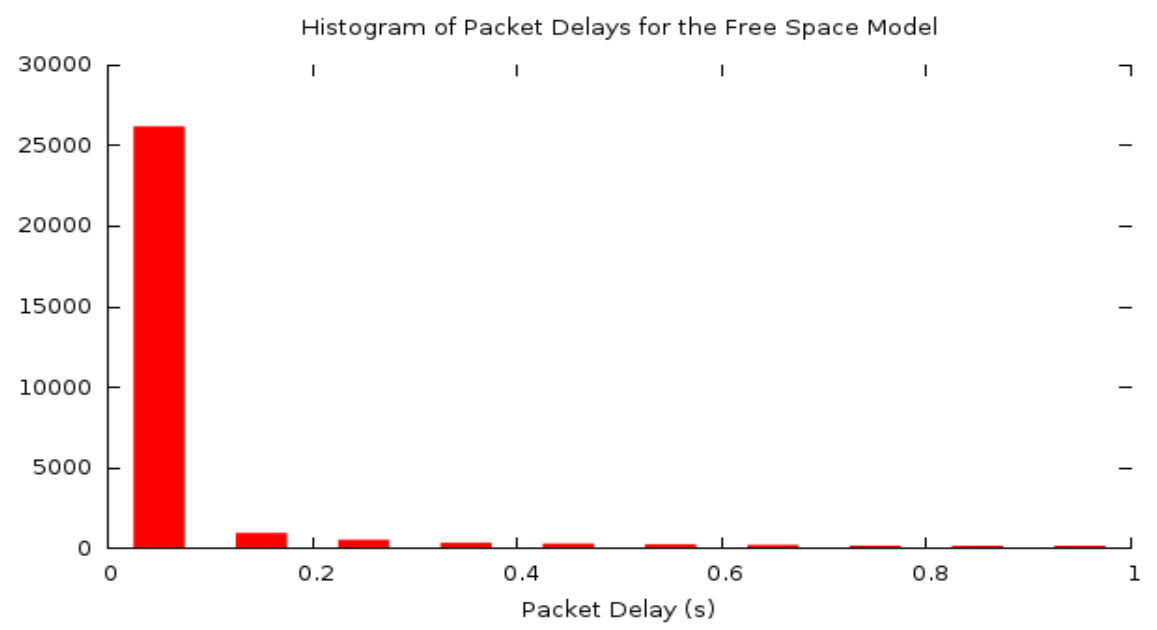

Figure 4. The histogram of the packet delays for the free space loss model

As also shown in Figures 2 and 3, the flow throughput trend and the packet loss rate trend are consistent for all three propagation models. The average flow throughput and the average packet loss rate are similar for the free space propagation model and the two-ray ground propagation model in all the cases of node density.

However, the average flow throughput for the shadowing model is significantly lower as compared with that of the other two models. The difference can reach about thirty percent when the node density is low. When the node density is high, however, the difference is smaller because the network becomes very congested in such a case and lots of packets are dropped for all the models.

Similar observations can be made in Figure 3 for packet losses for the three models. When the node density is low, the difference between the packet loss rates for the shadowing model and the other two models is big. When the node density becomes higher, however, the difference becomes smaller. The flow throughput performance and the packet loss rate performance thus corroborate each other in this case too.

The packet delay data shown in Figures 4 to 6 for the case of ten nodes also corroborate the results shown in earlier figures for the three models. As shown by Figures 4 to 6 , the distributions of packet delays are similar for the free space propagation model and the two-ray ground model. For these two models, most packets have a delay within 0.2 second. For the shadowing model, however, much more packets have a delay beyond 0.2 second. 


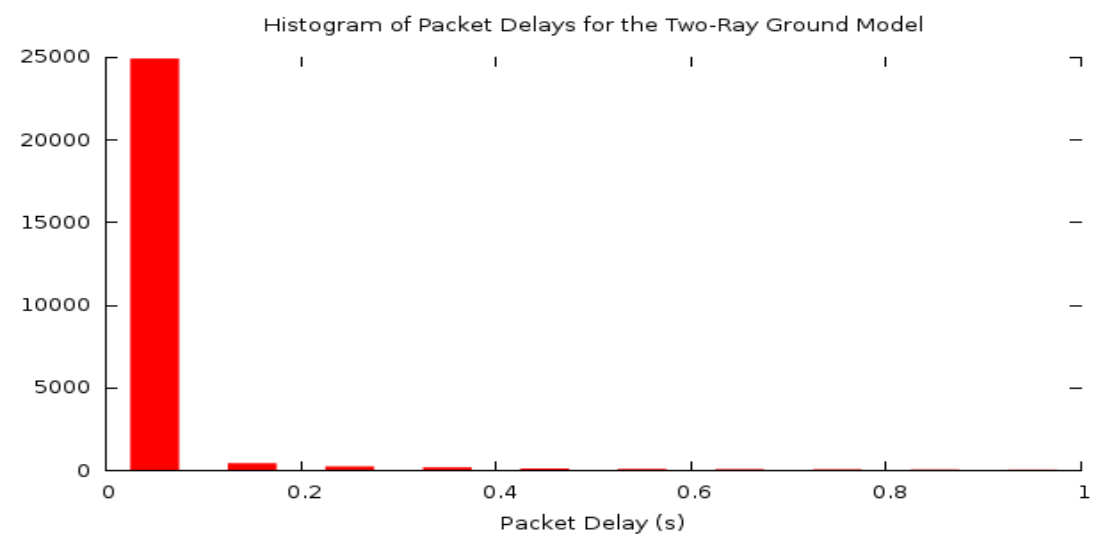

Figure 5. The histogram of the packet delays for the two-ray ground loss model

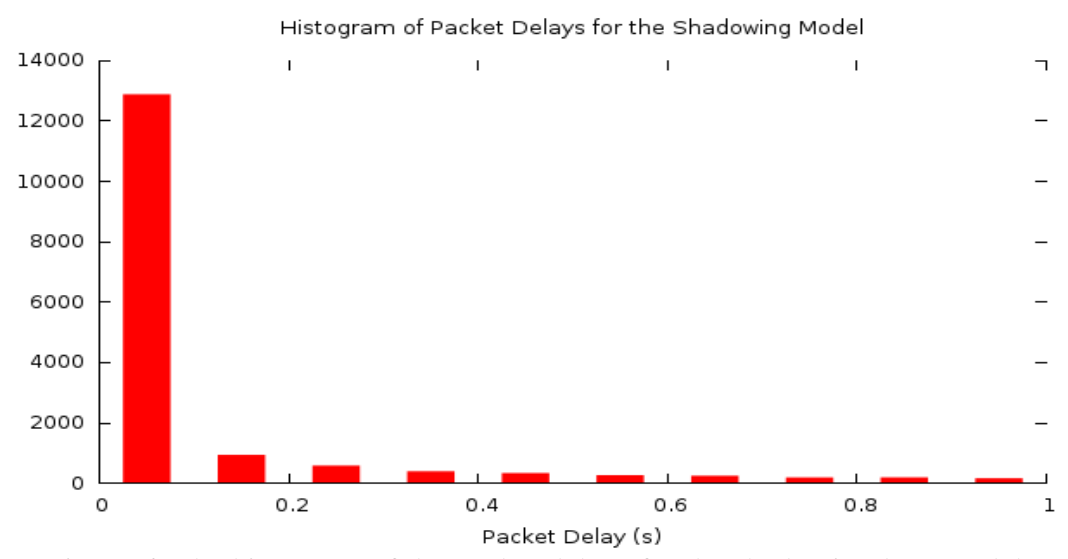

Figure 6. The histogram of the packet delays for the shadowing loss model

In summary, the choice of one of the three radio propagation models that are available in the ns2 network simulation package does have significant impact on simulation results for UAV networks. The differences between the results for the free space propagation model and the tworay ground model are small. The differences are more dramatic between the results for these two models and the shadowing model. The good news is, however, that even the shadowing model has simulation results that are very different from those of the other two models in each specific case of simulation, the trend of the simulation results when observed as a whole does show consistency for all three models.

\section{Conclusions}

We investigated the three radio propagation models that are available in the ns2 network simulation package for UAV networks. Our investigation shows that the shadowing model may generate simulation results that are significantly different from those of the free space loss model and the two-ray ground model in each specific case of simulation. Particularly, the flow throughput, the packet loss rate, and the packet delays consistently show much lower performance for the shadowing model than for the other two models. However, our results also show that when the simulation results are observed as a whole, the trend of flow throughput, packet loss rate, and 
packet delays is consistent for all three models. In conclusion, the choice of a radio propagation model in simulating UAV networks does have significant impact on the simulation results for each individual case of simulation, but such a choice does not affect the interpretation of the simulation data when performance trend is the only concern of investigation.

\section{ACKNOWLEDGEMENTS}

The authors would like to thank everyone who offered support to this project and the publication of its results.

\section{REFERENCES}

[1] T. H. Cox, C. J. Nagy, M. A. Skoog, and I. A. Somers, "Civil UAV Capability Assessment," NASA, 2004.

[2] M. Asadpour, D. Giustiniano, K. A. Hummel, and S. Heimlicher, "Characterizing 802.11N Aerial Communication," in Proceedings of the Second ACM MobiHoc Workshop on Airborne Networks and Communications, 2013, pp. 7-12.

[3] Y. Cai, F. Yu, J. Li, Y. Zhou, and L. Lamont, "Medium Access Control for Unmanned Aerial Vehicle (UAV) Ad-Hoc Networks With Full-Duplex Radios and Multipacket Reception Capability," Vehicular Technology, IEEE Transactions on, vol. 62, pp. 390-394, Jan 2013.

[4] A. Ollero, M. Bernard, M. La Civita, L. van Hoesel, P. Marron, J. Lepley, and E. de Andres, "AWARE: Platform for Autonomous self-deploying and operation of Wireless sensor-actuator networks cooperating with unmanned AeRial vehiclEs," in Safety, Security and Rescue Robotics, 2007, IEEE International Workshop on, Sept 2007, pp. 1-6.

[5] A. Alshbatat and L. Dong, "Adaptive MAC protocol for UAV communication networks using directional antennas," in Networking, Sensing and Control (ICNSC), 2010 International Conference on, April 2010, pp. 598-603.

[6] A. Alshbatat and L. Dong, "Cross layer design for mobile Ad-Hoc Unmanned Aerial Vehicle communication networks," in Networking, Sensing and Control (ICNSC), 2010 International Conference on, April 2010, pp. 331-336.

[7] D. Gu, G. Pei, H. Ly, M. Gerla, and X. Hong, "Hierarchical routing for multi-layer ad-hoc wireless networks with UAVs," in MILCOM 2000, vol. 1, 2000, pp. 310-314.

[8] D. Gu, H. Ly, X. Hong, M. Gerla, G. Pei, and Y.-Z. Lee, "C-ICAMA, a centralized intelligent channel assigned multiple access for multi-layer ad-hoc wireless networks with UAVs," in Wireless Communications and Networking Conference, 2000, IEEE, vol. 2, 2000, pp. 879-884.

[9] H. T. Friis, "A note on a simple transmission formula," in Proceedings of the I.R.E. and Waves and Electrons, 1946, pp. 254-256.

[10] T. S. Rappaport, "Wireless Communications, Principles and Practice," Prentice Hall, 1996.

[11] D. B. Johnson, D. A. Maltz, and Y.-C. Hu, "The Dynamic Source Routing Protocol for Mobile Ad Hoc Networks (DSR)," IETF Interet draft,draft-ietf-manet-dsr-10.txt, July 2004.

\section{Authors}

Jun Peng received his Ph.D. degree from the Department of Electrical, Computer \& Systems Engineering at Rensselaer Polytechnic Institute, Troy, New York, United States. He is currently an associate professor at the Electrical Engineering Department of University of Texas - Rio Grande Valley, Edinburg, Texas, United States. His research interests are in computer and communication networks. 